\title{
Identification of I-A $\beta$-Chain Residues Critical for T Cell Recognition of Peptide Antigens ${ }^{1}$
}

\author{
Thomas R. Esch, ${ }^{2}$ David J. MCKean, ${ }^{*}$ AND DaVID W. ThOMAS ${ }^{3}$ \\ Department of Microbiology and Immunology, University of Michigan Medical School, Ann Arbor, \\ Michigan 48109, and *Department of Immunology, Mayo Clinic, Rochester, Minnesota 55905
}

Received April 17, 1989; accepted July 6, 1989

\begin{abstract}
Major histocompatibility complex (MHC)-restricted recognition of antigen by T lymphocytes involves the formation of a complex composed of the $\mathrm{T}$ cell receptor, antigen, and restricting MHC molecule. To elucidate the interactions occurring within the antigen recognition complex, we have evaluated the ability of a panel of cell lines expressing mutated $\mathrm{I}-\mathrm{A}^{\mathrm{k}}$ molecules to function in the recognition by $T$ hybridoma cells of two distinct peptide antigens. Our results indicate that while alterations along the entire length of the proposed helical structure in the carboxyterminal half of the $\beta_{1}$ domain interfere with the I-A ${ }^{\mathrm{k}}$-restricted recognition of human fibrinopeptide $B$, mutations which affect recognition of hen egg lysozyme/I-A $A^{k}$ fall almost exclusively in the central portion of the helix. On the basis of these and previous results, we propose a "T cell receptor-mediated peptide exchange model" for formation of the antigen recognition complex. O 1989 Academic Press, Inc.
\end{abstract}

\section{INTRODUCTION}

Antigen-specific activation of helper $T$ lymphocytes requires the corecognition by the $\mathrm{T}$ cell receptor [TCR $]^{4}$ of the antigen and a specific class II MHC (Ia) molecule (reviewed in (1)). This "dual-recognition" process has multiple implications for the development and functions of the immune system and has thus been the focus of a large volume of research. A number of models for MHC-restricted antigen recognition have been advanced over the last several years (2-7), with the consensus revolving around the idea of a three-membered antigen recognition complex composed of the TCR, antigen, and restricting MHC molech les. The exact relationships among and interactions between these three elements, however, have yet to be detailed, even for a single specific case. Given our present understanding of the structures of certain antigenic peptides $(8,9)$ and of MHC molecules $(10-12)$, the elucidation of these relationships is perhaps best approached by focusing on antigen $[\mathrm{Ag}]$ and $\mathrm{MHC}$ and

\footnotetext{
${ }^{1}$ This research was supported by Grant IM-464 from the American Cancer Society (D.W.T.) and Grant CA26297 from NIH (D.J.M.). T.R.E. was supported by NIH National Research Service Award 1-T32GM-07544-09 from the National Institutc of Gencral Medical Sciences and by the Arthur Thurnau Trust Fund.

${ }^{2}$ Current address: The Wistar Institute, 36th Street at Spruce, Philadelphia, PA 19104.

${ }^{3}$ Current address: Biogen, 14 Cambridge Center, Cambridge, MA 02142.

${ }^{4}$ Abbreviations used: TCR, T cell receptor for antigen/MHC; MHC, major histocompatibility complex; $\mathrm{Ag}$, antigen; hFPB, human fibrinopeptide $\mathrm{B} ; \mathrm{HEL}$, hen egg lysozyme; APC, antigen-presenting cell(s).
} 
treating the combination of the two as the ligand for the TCR. Precise definition of such a TCR ligand and the Ag-MHC interaction that forms it would aid considerably our understanding of MHC-restricted antigen recognition.

In the present study, we have used a panel of transfectants expressing I- $A^{k}$ genes with mutations in the $\beta_{1}$ domain to identify amino acid residues in that domain which are important for recognition of two I-A ${ }^{k}$-restricted antigens, human fibrinopeptide $\mathrm{B}$ [hFPB] and hen egg lysozyme [HEL]. HEL in many ways represents what has come to be thought of as typical of protein antigens: the actual antigen in this case is a peptide fragment, minimally encompassing residues 52-61 of the protein, which is produced via intracellular "processing" of HEL by antigen-presenting cells [APC] (13) and has been shown to bind specifically to isolated I-A $\mathrm{A}^{\mathrm{k}}$ molecules in detergent solution (14), and which can be recognized by HEL-specific T cells on APC that have been pulsed with the whole protein or peptide for short periods and washed prior to exposure to the $T$ cells (15). hFPB, on the other hand, will stimulate hFPB-specific $T$ cells only when soluble antigen is present in the T cell-APC coculture and does not appear to bind to I- $A^{\mathrm{k}}$ in the absence of $\mathrm{T}$ cells $(16,19)$, but does bind specifically to the TCR on an hFPB-responsive T hybridoma in the absence of Ia (T. R. Esch and $D$. W. Thomas, manuscript in preparation). Taken together, the two systems represent a range of possibilities concerning the formation of and relationships within the antigen recognition complex of TCR, Ia, and antigen. Since the antigenic fine specificities are known in both cases $(9,17,18)$, defining the critical residues of the I- $\mathrm{A}^{\mathrm{k}}$ molecule for recognition of each antigen will greatly clarify our conception of the two TCR ligands.

\section{MATERIALS AND METHODS}

Cell lines. The $\mathrm{T}$ cell hybridoma 14E6.8.2, specific for $\mathrm{hFPB} / \mathrm{I}-\mathrm{A}^{\mathrm{k}}$, has been described previously (19). 3A9, specific for HEL (46-61)/I-A ${ }^{\mathrm{k}}(13)$, was a gift from Dr. Paul Allen, Washington University (St. Louis, MO). The B cell hybrid TA3 (20) was a generous gift from Dr. Laurie Glimcher, Harvard University (Boston, MA). Most of the APC lines expressing mutated I- $\mathrm{A}^{\mathrm{k}}$ genes have been described previously (21); further members of this series were similarly produced and maintained.

Antigens. hFPB (1-14) was obtained from Bachem (Torrance, CA). HEL (whole protein, Sigma Grade I) was purchased from Sigma (St. Louis, MO).

$T$ cell stimulation assay. T hybridoma cells $\left(5 \times 10^{4}-2 \times 10^{5}\right)$ were cocultured with APC $\left(1 \times 10^{4}-1 \times 10^{5}\right)$ and graded doses of antigen in microtiter wells (total volume $200 \mu \mathrm{l})$ for $24 \mathrm{hr}$. Triplicate aliquots of supernatant fluids were then removed and frozen for $\geqslant 24 \mathrm{hr}$ at $-70^{\circ} \mathrm{C}$. CTLL-2 cells $\left(4 \times 10^{3}\right)$ (a generous gift from Dr. David Steinmuller, University of Michigan) were added to thawed supernatant samples to give a final supernatant dilution of $1: 2$, cultured for $24 \mathrm{hr}$, and pulsed with $1 \mu \mathrm{Ci}\left[{ }^{3} \mathrm{H}\right]-$ thymidine for a further 6-12 hr. All wells were then harvested onto glass fiber filters using a Titer-Tek multiwell harvesting device (Skatron), and $\left[{ }^{3} \mathrm{H}\right]$ thymidine incorporation was assessed by liquid scintillation counting. Each data point presented represents the average cpm incorporated in the triplicate cultures, less the average incorporation measured when no antigen was added to the primary culture.

\section{RESULTS}

Expression of mutant $I-A^{k}$ molecules on $A P C$. The APC used in these experiments were created by transfection of mutant I- $\mathrm{A}_{\beta}^{\mathrm{k}}$ genes, produced by site-directed muta- 


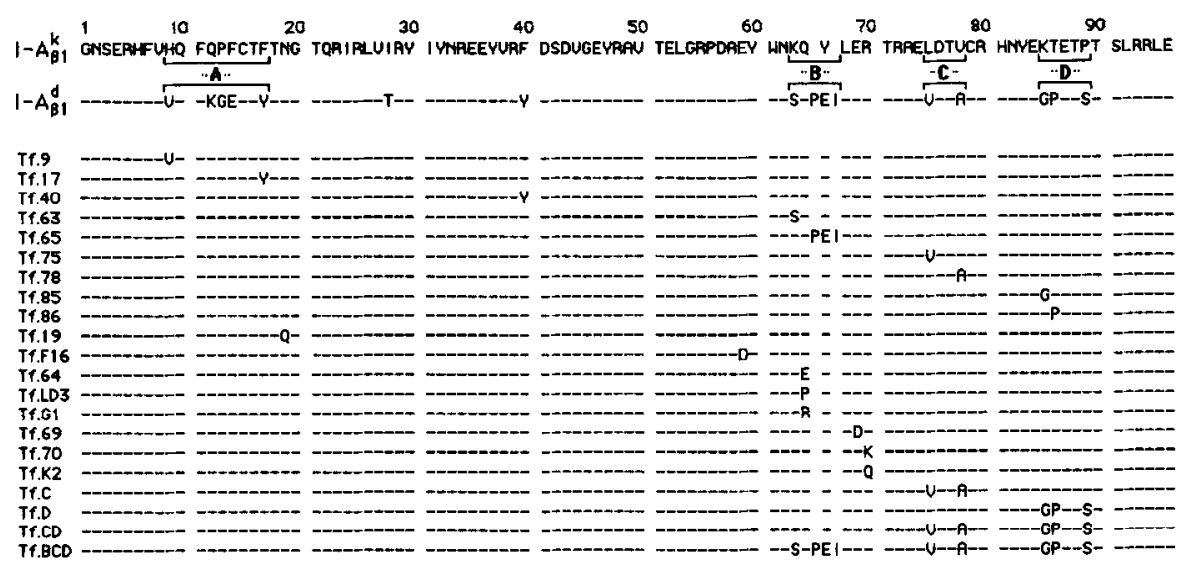

FIG. 1. Amino acid sequences of the $\beta_{1}$ domains of $I-A^{k}$ and $I-A^{d}$ and the mutants used in the experiments described in this paper. Dashes indicate the presence of amino acid residues corresponding to the wildtype I-A ${ }^{k}$ molecule.

genesis, along with wild-type $\mathrm{I}-\mathrm{A}_{\alpha}^{\mathrm{k}}$ genes into the B lymphoma line M12.C3, as described previously (21). The amino acid sequences of the $\beta_{1}$ domains of the wild-type $\mathrm{I}-\mathrm{A}^{\mathrm{k}}$ and $\mathrm{I}-\mathrm{A}^{\mathrm{d}}$ molecules are shown in Fig. 1 , along with the sequences of the I-A ${ }^{\mathrm{k}}$ mutants examined. Figure 2 shows the approximate positions of the altered residues within the structure proposed for the I-A molecule (12). The clones used were selected for expression of altered I- $\mathrm{A}^{\mathrm{k}}$ at levels similar to those of the $\mathrm{B}$ hybridoma TA3, which expresses single copies of the wild-type I-A ${ }^{\mathrm{k}}$ genes (20). Consistency of expression at

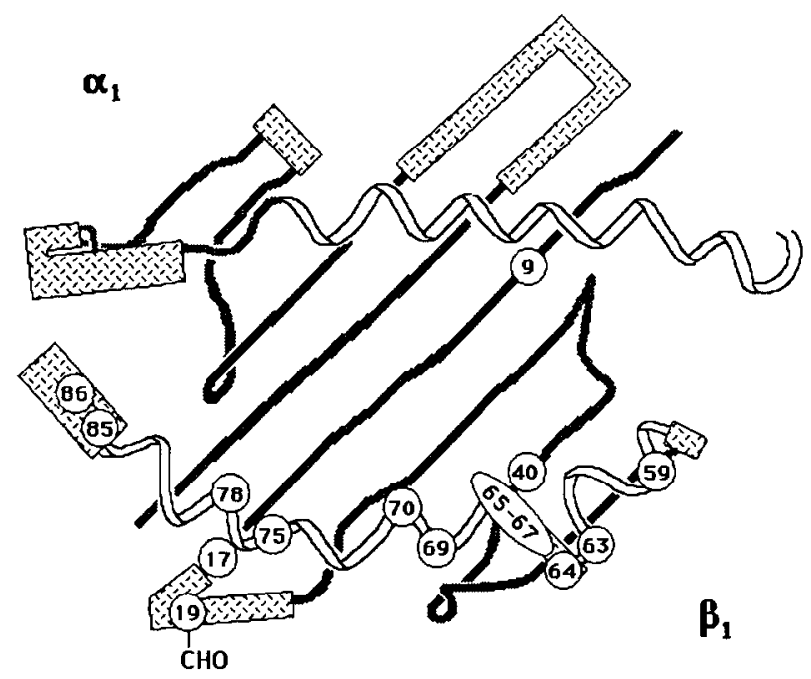

FIG. 2. Approximate positions in the proposed structure of the $\mathrm{I}-\mathrm{A}^{\mathrm{k}}$ molecule of the amino acid residues altered in the APC used in this investigation. In this representation, adapted from Ref. (12), the molecule is viewed from the TCR's point of view, looking toward the APC surface from directly above the putative peptide binding site. The $\mathrm{N}$-linked glycosylation site shown here is destroyed when residue 19 is mutated (Tf.19). 


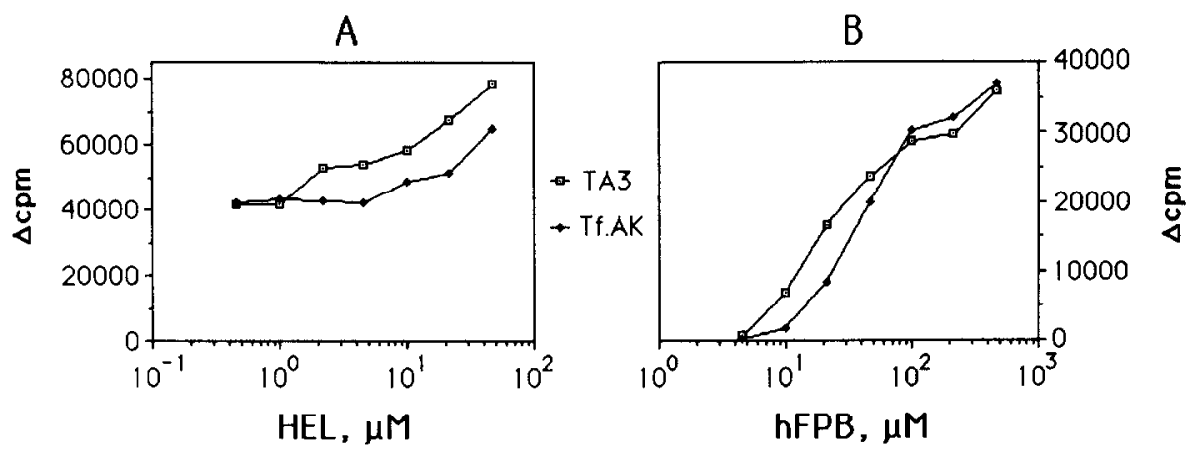

FIG. 3. Responses of I-A $A^{k}$-restricted T hybridomas to antigen in the presence of APC expressing wildtype I-A $\mathrm{A}^{\mathrm{k}} \mathrm{T}$ cells $\left(10^{5}\right)$ were cultured in microtiter wells with varying doses of the appropriate antigen and $5 \times 10^{4}$ TA3 (I-A ${ }^{k}$ expressing B hybridoma) or Tf.A.K (M12.C3 B lymphoma transfected with I-A ${ }^{k} \alpha-$ and $\beta$-chain genes) cells as APC, as indicated; culture supernatants were then tested for IL- 2 content by their ability to support proliferation of CTLL-2 cells as measured by uptake of tritiated thymidine. (A) Response of the HEL (46-61)-specific T hybrid 3A9; (B) response of the hFPB-specific T hybrid 14E6.8.2. This figure shows data from one representative experiment.

this level was verified by flow cytometric analysis of selected APC at intervals during the course of these investigations (data not shown). A clone expressing wild-type I$\mathrm{A}^{\mathrm{k}} \alpha$ - and $\beta$-chain genes, designated Tf.A.K, can present both hFPB and HEL to two $\mathrm{T}$ hybridomas, $3 \mathrm{~A} 9$ and 14E6.8.2, which respond to HEL (46-61) and hFPB, respectively, in the context of $\mathrm{I}-\mathrm{A}^{\mathrm{k}}$ (Fig. 3), showing that processing (for HEL) and association of antigen with Ia occur normally in such transfectants. Any differences in the abilities of the various APC thus cannot be attributed to differences in Ia density, antigen processing, or any other APC-mediated processes, but should reflect the inherent qualities of the mutant molecules.

Functional analysis of regional mutations using $h F P B$ - and HEL-specific Thybridomas. The polymorphic amino acid residues within the $\beta_{1}$ domain of the I-A molecule can be grouped into four polymorphic regions which have been designated -A . . . . B . . . C $\cdots$, and . . . . ((21) see Fig. 1). Transfectants expressing I-A $\beta$ chain genes constructed to encode residues characteristic of the I- $A^{d}$ molecule in one or more of these regions were tested for their ability to function in the antigen-specific responses of $3 \mathrm{~A} 9$ and 14E6.8.2. Each $\mathrm{T}$ hybrid was cultured with graded doses of the appropriate antigen, with each of the cloned transfectants in turn serving as APC. T cell stimulation was then evaluated in terms of secretion of IL-2 into the culture supernatant. Figure 4 shows the dose-response curves exhibited by $3 A 9$ (A) and 14E6.8.2 (B) using Tf.C, Tf.D, Tf.CD, Tf.BCD, and TA3 as APC. From these results, it appears that the entire carboxy-terminal portion of the $\beta_{1}$ domain is critical for recognition of hFPB by $14 \mathrm{E} 6.8 .2$. In contrast, only the $\ldots \mathrm{B} \cdots$ and $\cdots \mathrm{C} \cdot$ regions are essential for recognition of HEL, as the alteration of the entire . D . segment has no effect on the response of $3 \mathrm{~A} 9$.

Functional analysis of single residue changes. The preceding analysis was refined and expanded by examining the functional capacity of 17 APC clones expressing I$\mathrm{A}^{\mathrm{k}}$ molecules with single amino acid changes in the $\beta_{1}$ domain. Nine of these 17 mutants represent changes at polymorphic residues from the $k$ toward the $d$ haplotype; the remainder include changes at these same positions to other amino acids and 


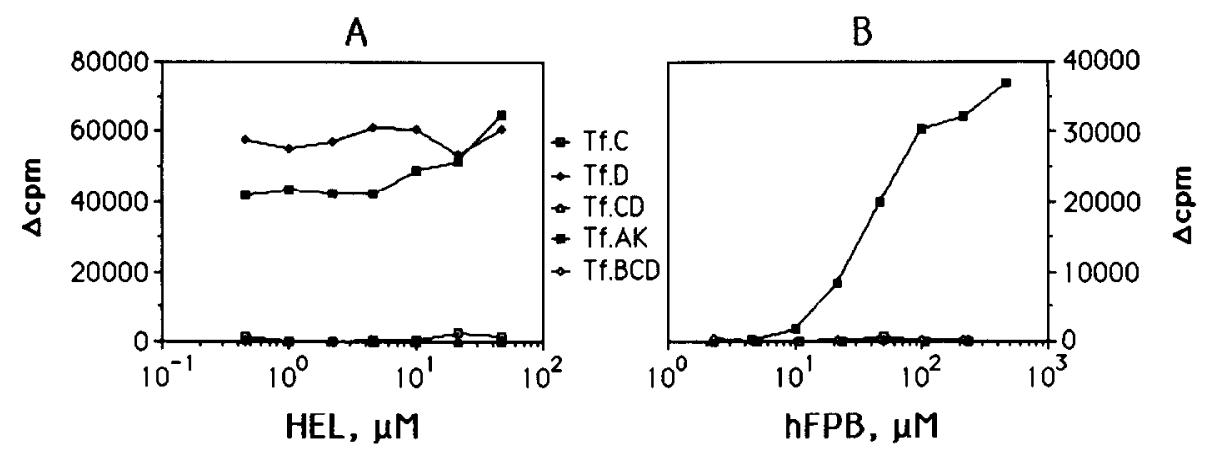

FIG. 4. Responses of $I-A^{k}-$ restricted $T$ hybridomas to antigen in the presence of APC expressing I-A ${ }^{k}$ molecules with regional alterations. (A) $5 \times 10^{4} 3 \mathrm{~A} 9 / 6$ and $5 \times 10^{4} \mathrm{APC}$ or (B) $10^{5} 14 \mathrm{E} 6.8 .2$ and $10^{5} \mathrm{APC}$ were cultured in microtiter wells with varying doses of HEL or hFPB, respectively; culture supernatants were then tested for IL-2 content as described above. Data from a single representative experiment are shown in this figure; the results of multiple experiments are summarized in Fig. 6.

alterations in nonpolymorphic residues (see Fig. 1). Typical dose-response curves using four different APC types with 3A9 and 14E6.8.2 are shown in Figs. 5A and 5B, respectively. The responses obtained with each transfectant are referenced to those obtained with Tf.A.K, the transfectant expressing wild-type I-A ${ }^{\mathrm{k}}$. Tf.40 $\left(\mathrm{Phe}^{40} \rightarrow \mathrm{Tyr}\right)$ plus antigen thus stimulates both $\mathrm{T}$ hybrids as effectively as cells bearing unaltered I$\mathrm{A}^{\mathrm{k}}$, Tf.86 $\left(\mathrm{Thr}^{86} \rightarrow \mathrm{Pro}\right)$ is somewhat less effective than wild-type in presentation of HEL to $3 \mathrm{~A} 9$, and Tf.9 $\left(\mathrm{His}^{9} \rightarrow \mathrm{Val}\right.$ ) plus hFPB gives only weak stimulation of 14E6.8.2. Tf.K2 $\left(\mathrm{Arg}^{70} \rightarrow \mathrm{Gln}\right)$ does not function in recognition of either antigen. The results for all 17 transfectants bearing single amino acid changes are summarized in Fig. 6.

\section{DISCUSSION}

Considerable advances have been made in recent years toward defining the mechanism(s) of MHC-restricted antigen recognition by $\mathrm{T}$ lymphocytes. The accumulated
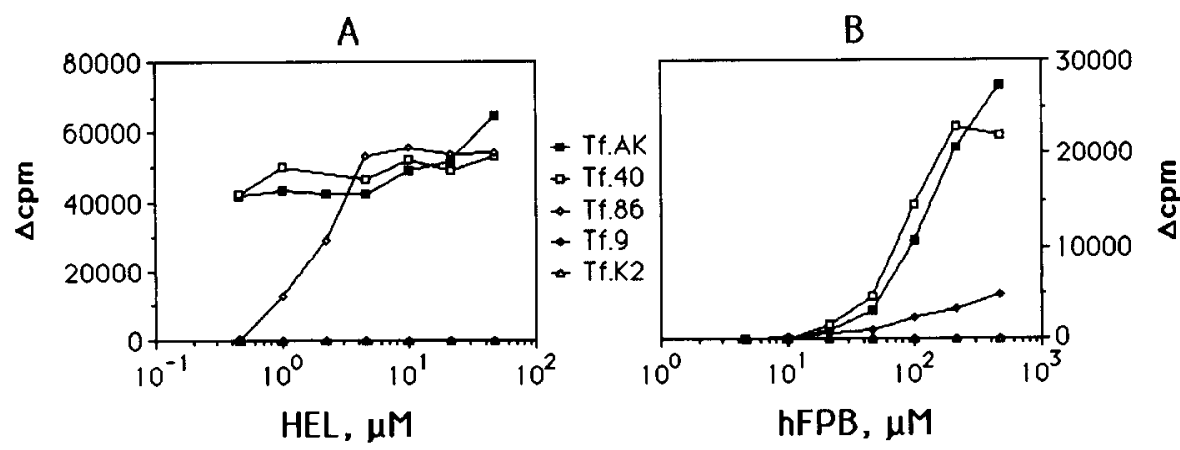

FIG. 5. Responses of $I-A^{k}$-restricted $T$ hybridomas to antigen in the presence of APC expressing I-A ${ }^{k}$ molecules with single amino acid substitutions. (A) $5 \times 10^{4} 3 \mathrm{~A} 9 / 6$ and $5 \times 10^{4} \mathrm{APC}$ or (B) $10^{5} 14 \mathrm{E} 6.8 .2$ and $10^{5} \mathrm{APC}$ were cultured in microtiter wells with varying doses of HEL or hFPB, respectively; culture supernatants were then tested for IL-2 content as described above. Data from a single representative experiment are shown in this figure; the results of multiple experiments are summarized in Fig. 6. 

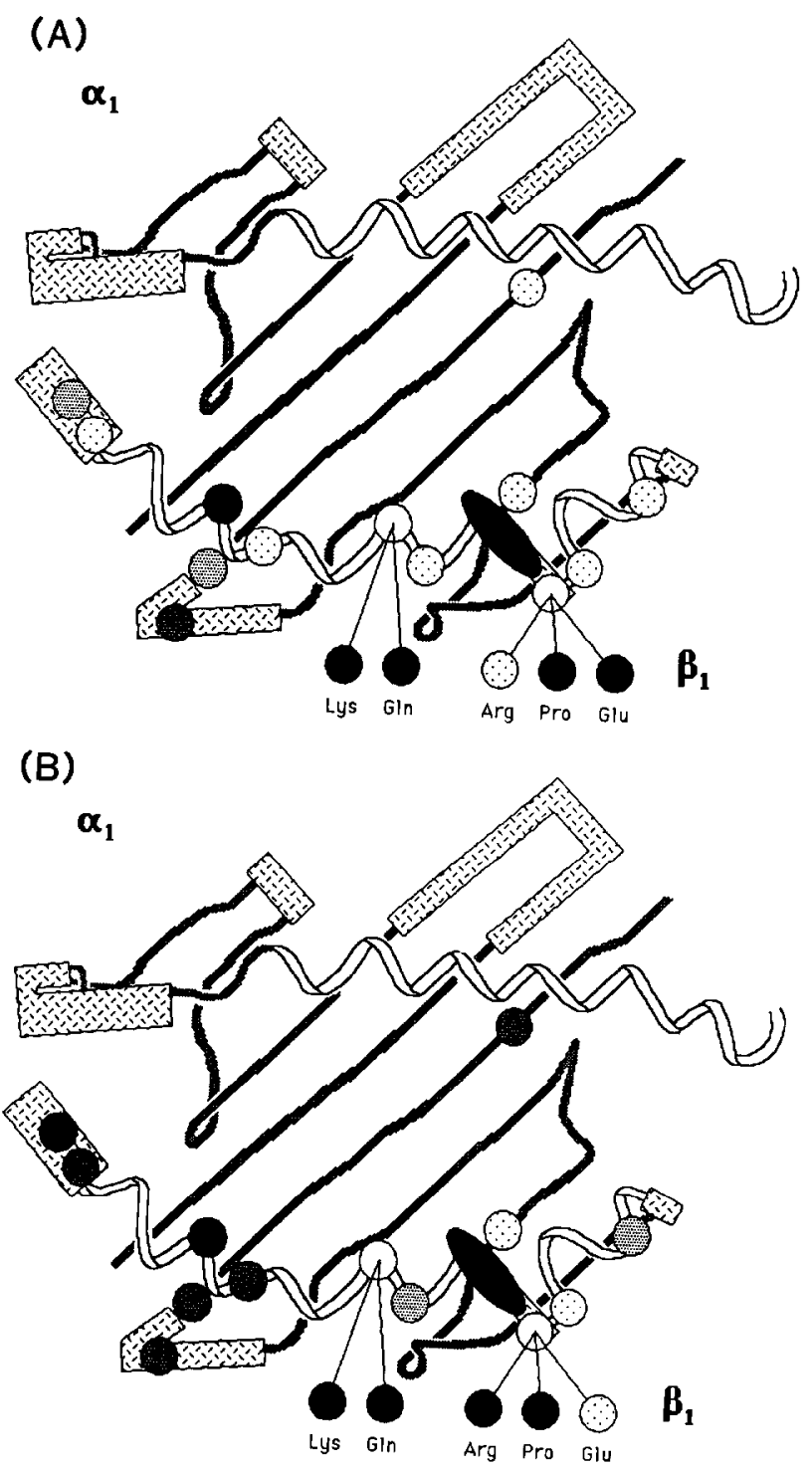

FIG. 6. Summary of responses of I-A $A^{k}$-restricted T hybridomas to antigen in the presence of APC expressing $\mathrm{I}-\mathrm{A}^{\mathrm{k}}$ molecules with single amino acid substitutions. (A) $5 \times 10^{4} 3 \mathrm{~A} 9 / 6$ and $5 \times 10^{4} \mathrm{APC}$ or (B) $10^{5}$ 14E6.8.2 and $10^{5} \mathrm{APC}$ were cultured in microtiter wells with varying doses of HEL or hFPB, respectively; culture supernatants were then tested for IL-2 content as described above. The degree of stimulation achieved with $I-A^{k}$ mutated at each position is indicated by shading of the circle at that position in the model structure: $(\bigcirc)$ T cell stimulation equal to that achieved with cells expressing wild-type I-A ${ }^{k}$ (TA3 or Tf.A.K cells); (-) a somewhat weaker response, generally reaching a full stimulation at higher antigen concentrations; (-) a weak response, not achieving full IL-2 production even at the highest antigen concentration tested; and ( $)$ a lack of any $T$ cell response at any concentration of antigen. These results are based on three to six independent trials with each antigen-APC-T cell combination.

results of a wide variety of experimental approaches have led to widespread acceptance of the idea that such recognition involves the formation of a complex including, at a minimum, the TCR, restricting MHC molecule, and antigen. Controversy re- 
mains, however, as to how this complex is formed and, in particular, how its elements relate to one another. In a few systems, notably those utilizing peptide antigens from cytochrome $c(8)$ and hen egg lysozyme (9), the role played by each amino acid residue of the antigen has been examined, allowing at least partial distinction of TCR-contact (epitope) from Ia-contact (agretope) sites on the antigen. Such results provide only a partial description of the ligand recognized by the TCR, and require a parallel analysis of the roles played by residues in the Ia molecule to complete the picture. In this report, we present the first portion of such an analysis, evaluating the relevance of mutations in the $\beta_{1}$ domain of I-A $\mathrm{A}^{\mathrm{k}}$ for presentation of two peptide antigens.

The two antigen recognition systems examined in the experiments described above exhibit several features in common with respect to the role of the Ia molecule, as well as a number of interesting differences. Regions $\cdots \mathrm{B} .$. and $. \mathrm{C} .$. of the I- $\mathrm{A}^{\mathrm{k}}$ molecule, encompassing residues 63-67 and 75-78, respectively, appear to be critical in the recognition of both hFPB and HEL. In the projected structural model for class II MHC molecules, these regions occupy roughly the middle of the helical section of the $\beta$ chain (12). Since any peptide large enough to serve as a $\mathrm{T}$ cell antigen would, when placed into the interhelical cleft, almost certainly interact with at least one of thesc rcgions, it scems likely that the $\cdots$ B. and.$C$. regions should be important for the recognition of any peptide antigen bound in the cleft of the Ia molecule. This is not to say that the critical residues within each region will be the same for all peptides. Changing one of the.$C$. region polymorphic residues, $\mathrm{Leu}^{75}$, to valine, for example, has much less effect on recognition of HEL than on recognition of hFPB.

In contrast to the role of the..$B$. and . . . . regions in recognition of both hFPB and HEL (46-61), the region designated . .D . appears to be important only in the hFPB system. The structure of this area is not clear from computer modeling (12), and it is difficult to predict what conformations may appear in the mutant molecules. The hFPB molecule may interact with $\mathrm{I}^{-\mathrm{A}^{\mathrm{k}}}$ in a largely extended conformation, requiring either interaction with . D . . region residues or a gap between the $\alpha$ - and the $\beta$-chain helices on that end of the cleft, either of which could be destroyed by the mutations examined. The HEL fragment produced by APC processing, on the other hand, may be as short as 10 residues (52-61) in a compact $\alpha$-helical configuration, as proposed by Allen et al. (9). This comparatively small fragment may not extend far enough down the cleft to involve the . . D . r region at all.

It is of interest that in several cases, the size of the amino acid side chain at a given position appears to be the most important factor, rather than the side chain's charge or hydrophobicity. Thus, changing Leu ${ }^{75}$ to valine, which has a similarly hydrophobic but slightly larger side chain, impairs recognition of hFPB by $14 \mathrm{E} 6.8 .2$, and replacing $\mathrm{Val}^{78}$ with alanine abrogates recognition of both hFPB and HEL. Substitutions for Gln ${ }^{64}$ have more complex effects along the same general lines: replacement with glutamic acid (similar in size, but negatively charged) does not significantly affect recognition of hFPB, but a change to arginine (larger and positively charged) results in loss of function in this system. It should be noted, however, that the inverse of this pattern is observed for the presentation of HEL to 3A9 by these two mutants. Since the side chain of residue 64 appears to be situated on the face of the helix away from the antigen binding cleft, the effects of various substitutions at this point are more likely to reflect TCR-Ia contacts, i.e., alterations in the histotope. Examination of the properties of a more comprehensive set of altered Ia molecules would be necessary to determine if this phenomenon is general in antigen-Ia and/or TCR-Ia interactions. 
One potential problem with studies of this type is that the effects of any given mutation on the overall structure of the molecule in question cannot always be reliably predicted. It could thus be argued that any alteration in an Ia molecule which changes its ability to function in presentation of a given antigen may do so as a result of some gross conformational disruption, rather than by modifying a specific Ag-Ia or TCR-Ia interaction. Our characterization of the recognition of two distinct antigens bypasses this objection in many cases by virtue of the fact that many mutant la species which function poorly in recognition of one antigen show normal function with the other. For example, Tf.64 (Gln ${ }^{64} \rightarrow$ Glu) is completely unable to present HEL to $3 \mathrm{~A} 9$, but functions normally in recognition of $\mathrm{hFPB}$, indicating that the molecule is present in a functional form which simply lacks the necessary contacts for the HEL/ 3A9 system. There are, of course, some instances in which significant conformational effects would be expected (e.g., Tf.LD3, Gln ${ }^{64} \rightarrow$ Pro) and in which loss of function is in fact observed, and other cases which show loss of function with both antigens, where this line of reasoning does not hold, although the results of antibody binding studies using these transfectants are in most cases not consistent with significant conformational changes (21). The instances in which neither of these observations applies are few in number and have little impact upon our primary conclusions.

The hypothetical structure for class II MHC molecules has been used as the basis for modeling the binding of HEL (52-61) within the cleft between the $\alpha_{1}$ - and $\beta_{1}$ domain helices (12). Although the experimental results described above do not provide a rigorous test of these models, certain of our observations may be of interest in this regard. The apparent importance of size, rather than charge or hydrophobicity, of amino acid side chains in the I- $\mathrm{A}^{\mathrm{k}}$ molecule may be relevant to the interaction proposed in one of the HEL (52-61) binding models between Asp ${ }^{52}$ of the HEL peptide and $\mathrm{Arg}^{70}$ of the $\mathrm{I}-\mathrm{A}^{\mathrm{k}} \beta_{1}$ helix. As demonstrated above, changing $\operatorname{Arg}^{70}$ to lysinc, which also possesses a large, positively charged side chain, abolishes the ability of the molecule to present HEL. Although this result might also reflect a necessary TCR interaction site, it tends to favor a model for binding of the HEL peptide which does not require a charged-pair interaction involving that particular position in the Ia molecule. A second point of particular interest is the hydrophobic interaction area postulated in both of the models for binding of HEL (52-61) to I-A $\mathrm{A}^{\mathrm{k}}$, involving $\mathrm{Ile}^{58}$ of the HEL peptide and $\mathrm{Phe}^{26}$ and Gly ${ }^{66}$ of the $\alpha_{1}$ domain. Our results indicate that $\mathrm{Val}^{78}$ of the I-A $\mathrm{A}^{\mathrm{k}} \beta_{1}$ domain is critical for presentation of HEL to $3 \mathrm{~A} 9$. This side chain, predicted to point into the antigen binding cleft, would appear to be involved in a hydrophobic Ag-Ia interaction, possibly with $\mathrm{Ile}^{58}$ of the HEL peptide, rather than in a direct contact with the TCR. The change from valine ( $k$ haplotype) to alanine ( haplotype) could weaken such an interaction and contribute to the inability of the I$A^{d}$ molecule to bind HEL (52-61) (16). The lesser effect observed in the presentation of HEL by Tf. 75 (changing Leu ${ }^{75}$ to valine) may have a similar basis. These residues may be part of a general hydrophobic interaction site, extending across the interhelical cleft, which interfaces with the hydrophobic segments common to most peptides which are antigenic for T cells (23) and to peptides which bind to isolated Ia molecules (24). Such an interaction site, varying subtly among haplotypes, could be at least partially responsible for the broad specificities observed for antigen-Ia interactions.

The data presented above, indicating that hFPB may occupy the putative antigenbinding cleft of the I-A ${ }^{k}$ molecule, must be reconciled with our previous observation that $\mathrm{I}-\mathrm{A}^{\mathrm{k}}$ expressing APC pulsed with $\mathrm{hFPB}$ in the absence of $\mathrm{T}$ cells cannot subse- 
quently stimulate 14E6.8.2 (19) and the fact that hFPB does not bind to isolated I$A^{k}(16)$. To explain the requirement for $T$ cell mediation of the $h F P B-I-A^{k}$ association, we propose that interaction of the TCR with Ia mediates or facilitates the movement of peptides into and out of the interhelical cleft in the Ia molecule. If the bound peptide has no affinity for the TCR, it can dissociate from the TCR-Ia complex, allowing another peptide to bind. If, on the other hand, the original occupant of the cleft is the "correct" antigen for the TCR, its avidity for the TCR-Ia complex will result in its retention and binding to the complex, potentially leading to activation of the T cell. This TCR-mediated peptide exchange mechanism may also facilitate binding of peptides to previously unoccupied Ia binding clefts, if such species exist on the cell surface. Peptides may be made available for the exchange process via preliminary association with accessory structures on the APC surface $(25,26)$ or, in the case of hFPB, with the TCR itself. In this regard, we have found that synthetic HEL (46-61) peptide, which binds rather strongly to isolated $I-A^{k}(14,16)$, nevertheless is unable to specifically interfere with the $\mathrm{I}-\mathrm{A}^{\mathrm{k}}$-restricted presentation of $\mathrm{hFPB}$ to $14 \mathrm{E} 6.8 .2$, even at 30-fold molar excess (T. R. Esch, unpublished results). This result is consistent with the TCR-mediated peptide exchange model described above. Antigenic peptides which have been found to bind to isolated la presumably are able to displace previously bound peptides by some $\mathrm{T}$ cell-independent mechanism which is ineffective for hFPB. The slow association rates found for these interactions, however, support the notion that functionally effective peptide binding to Ia may require either an active mechanism on the APC surface or a T-cell mediated process such as the peptide exchange mechanism proposed above to increase the rate of antigen-Ia complex formation into the range of potential physiological relevance. The extension of the exchange process to a cellular scale may thus help resolve the problems presented by the combination of a relatively short cell surface half-life for Ia molecules (27), slow peptide-Ia dissociation rates (28), and binding of self-derived peptides to Ia $(16,29)$. To further define this process, we are currently examining the effects of mutations in the I- $\mathrm{A}^{\mathrm{k}}$ molecule on the binding of $\mathrm{hFPB}$ to its TCR and consequent formation of potential antigen recognition complexes.

\section{ACKNOWLEDGMENT}

The authors thank Dr. Paul Allen for providing the T hybridoma 3A9 and HEL (46-61) peptide and for his helpful comments.

\section{REFERENCES}

1. Schwartz, R. H., In "Fundamental Immunology" (W. E. Paul, Ed.), pp. 379-438. Raven Press, New York, 1984.

2. Heber-Katz, E., Hansburg, D., and Schwartz, R. H., J. Mol. Cell. Immunol. 1, 3, 1983.

3. Ashwell, J. D., and Schwartz, R. H., Nature (London) 320, 176, 1986.

4. Zinkernagel, R. M., and Doherty, P. C., Nature (London) 251, 547, 1974.

5. Sherman, L. A., Nature (London) 297, $511,1982$.

6. Schwartz, R. H., Annu. Rev. Immunol. 3, 327, 1985.

7. Cleveland, W. L., and Erlanger, B. F., Mol. Immunol. 21, 1037, 1984.

8. Fox, B. S., Chen, C., Fraga, E., French, C. A., Singh, B., and Schwartz, R. H., J. Immunol. 139, 1578, 1987.

9. Allen, P. M., Matsueda, G. R., Evans, R. J., Dunbar, J. B., Jr., Marshall, G. R., and Unanue, E. R., Nature (London) 327, 71 3, 1987. 
10. Bjorkman, P. J., Saper, M. A., Samraoui, B., Bennett, W. S., Strominger, J. L., and Wiley, D. C., Nature (London) 329, 506, 1987.

11. Bjorkman, P. J., Saper, M. A., Samraoui, B., Bennett, W. S., Strominger, J. L., and Wiley, D. C., Nature (London) 329, 512, 1987.

12. Brown, J. H., Jardetzky, T., Saper, M. A., Samraoui, B., Bjorkman, P. J., and Wiley, D. C., Nature (London) 332, 845, 1988.

13. Allen, P. M., Strydom, D. J., and Unanue, E. R., Proc. Natl. Acad. Sci. USA 81, 2489, 1984.

14. Babbitt, B. P., Allen, P. M., Matsueda, G., Haber, E., and Unanue, E. R., Nature (London) 317, 359, 1985 .

15. Allen, P. M., and Unanue, E. R., J. Immunol. 132, $1077,1984$.

16. Babbitt, B. P., Matsueda, G., Haber, E., Unanue, E. R., and Allen, P. M., Proc. Natl. Acad. Sci. USA 83, 4509, 1986.

17. Thomas, D. W., Hsieh, K.-H., Schauster, J. L., Mudd, M. S., and Wilner, G. D., J. Exp. Med. 152, $620,1980$.

18. Peterson, L. B., Wilner, G. D., and Thomas, D. W., J. Immunol. 130, 2542, 1983.

19. Thomas, D. W., and Solvay, M. J., J. Immunol. 137, $3401,1986$.

20. Glimcher, L. H., Hamano, T., Asofsky, R., Sachs, D. H., Pierres, M., Samelson, L. E., Sharrow, S. O., and Paul, W. E., J. Immunol. 130, 2287, 1983.

21. Buerstedde, J.-M., Pease, L. R., Bell, M. P., Nilson, A. E., Buerstedde, G., Murphy, D., and McKean, D. J., J. Exp. Med. 167, 473, 1988.

22. Oi, V. T., Jones, P. P., Goding, J. W., Herzenberg, L. A., and Herzenberg, L. A., Curr. Top. Microbiol. Immunol. 81, 115, 1978.

23. Rothbard, J. B., and Taylor, W. R., EMBO J. 7, 93, 1988.

24. Sette, A., Buus, S., Colon, S., Miles, C., and Grey, H. M., J. Immunol. 141, 45, 1988.

25. Lakey, E. K., Margoliash, E., and Pierce, S. K., Proc. Natl. Acad. Sci. USA 84, 1659, 1987.

26. Nairn, R., Spengler, M. L., Hoffman, M. D., Solvay, M. J., and Thomas, D. W., J. Immunol. 133, $3225,1984$.

27. Emerson, S. G., Murphy, D. B., and Cone, R. E., J. Immunol. 125, 406, 1980.

28. Buus, S., Sette, A., Colon, S. M., Jenis, D. M., and Grey, H. M., Cell 47, 1071, 1986.

29. Lorenz, R. G., and Allen, P. M., Proc. Natl. Acad. Sci. USA 85, 5220, 1988. 